\title{
Service de détection des avalanches au chantier des Brévières*
}

\author{
The avalanche forecasting department \\ of the Brévières construction site
}

\author{
PAR M. SALES \\ CHEF DE SERVICE DF DRTECTION HUS AVALANCHES
}

\begin{abstract}
Rappel des conditions de déclenchement des avalanches catastrophiques de ferrier 1950 au chantier des Brévieres près de Tignes. Mise sur pied d'un service de détection. -. Son organisation, ses méthodes, son équipement, son fonctionnement, son effeacite lors des avalanches du mois de fevier 1951
\end{abstract}

\begin{abstract}
Conditions existing at time of the catastrophic abalanches in Febratry, 1950 on the Brevières site near Tignes. Creation of an avalanche forecasting dept. Its organisation, methods, squipment, operation and its efficiency during the abalanches in February, 1951.
\end{abstract}

Qu'il me soit permis, avant d'entrer dans le sujet qui nous intéresse, de vous situer le chantier des Brévières, près de Tignes, en Savoie, et vous dire quelques mots sur les dangers d'avalanches qui le menacent.

L'agglomération des Brévières est à une altitude de $1.550 \mathrm{~m}$ et est desservie par la R.N. 202 de Bourg-Saint-Maurice, à Val-d'Isère.

Cette agglomération, primitivement composéc de maisons indigènes, s'est considérablement développée par suite de l'établissement de baraquements, chalets, ateliers, dortoirs, cantines, habitations particulieres pour les cadres, infirmerie, bureaux, etc., nécessités par la construction du grand barrage de Tignes. Il a donc fallu ramasser, sur une superficie malheureusement restreinte, toutes ces habitations, et bien que la sécurité dans l'implantation de ces habitations ait été assurée en grande partie, la nature à plusieurs reprises a déjoué les prévisions humaines.

L'agglomération des Brévières, fortement n-

* Confèrence faite à la Société Hydrotechnique de France. caissce au fond de la vallée, domince au nord-est par la cote 2600 et au sud par la cote 2800 , est menacée au sud par une avalanche en provenance de la cote 2200 dite avalanche de la Sache, puis par une avalanche en provenance de la cote 2300 , dite du bois de l'Ours, à l'emplacement actuel de la carrière des Brévières, au sud-est par l'avalanche du versant dit de la Davie, en provenance de la cote 2600. L'avalanche de la Sache était la plus à redouter cependant.

Sans compter les maisons anciennes du village et ses habitants, le chantier des Brévières comporte 164 bâtiments, tant ateliers qu'habitations proprement dites, qui englobent dans leurs murs une population de 2.760 personnes environ.

Ces constructions sont réparties sur une surface de $31.700 \mathrm{~m}^{2}$ à peu près. Comme on le voit, l'espace est assez réduit, mais il n'était guère possible de procéder autrement. Si je livre ces quelques chiffres, c'est pour vous montrer à quel point le problème est vaste et digne d'intérêt, et par là même, mérite qu'on s'y attarde. 
Ceci dit, comment et à la suite de quelles circonstances a-t-on décidé la création d'un Service de Prévision d'Avalanches?

\section{Accident du 10 février 1950}

Depuis quelques jours, la neige tombait sans arrêt, par un vent violent, et s'accumulait dans les combes, en équilibre instable : plus d'un mètre de neige seche en vingt-quatre heures.

Le 10 février 1950, à 7 h. 30 , une coulée de neige se produisail à proximité du couloir dit «de la Sache » (au sud) et s'abattait, en franchissant une route qui servit de tremplin, sur la cité dite «Cité des Ruines », faisant six victimes et rasant deux chalets, trois autres étant presque entièrement démolis.

A la suite de cet accident, les entreprises, notamment l'Entreprise Industrielle, en accord avec E.D.F., décidaient des mesures de précautions à prendre : une série d'évacuations étaient ordonnées.

Toute la nuit, la neige n'a cessé de tomber et de nombreuses coulées se produisirent.

Le 11 février, un autre accident était à déplorer sur le chantier de l'Entreprise Rossetti, travaillant à la construction du premier troncon de la R.N. 202, mise en service pour remplacer celle qui allait être immergée. Un chauffeur de Dodge était enseveli avec son véhicule sous une avalanche tombée entre deux tunnels, sur la route allant à Val-d'Isère.

Dans l'après-midi, les représentants des entreprises intéressées venaient visiter les lieux de l'accident des Ruines et complétaient les mesures de sécurité déjà prises.

Dans la nuit du 12 février, une avalanche de plaque de neige se détachait à nouveau dans le couloir de la Sache et obstruait la route dite « des Boisses ». Il n'y eut heureusement aucun accident à déplorer, et cela grâce au dispositif de sécurité mis en vigueur.

Egalement le 12 février au matin, une avalanche en provenance de la carrière des Brévières emportait le silo et le tapis du concasseur primaire et les projetait dans l'Isère, et sur la rive opposée, couchait également quelques poteaux télégraphiques le long de la R.N. 202.

Dans la nuit du 12 au 13 février, par suite de conditions météorologiques satisfaisantes, le cicl s'éclaircissait, et le 13 au matin, on pouvait apercevoir la zone de rupture de l'avalanche de la Carrière des Brévières. Cette cassure avait une épaisseur d'environ $3 \mathrm{~m}$ de neige.

A 15 h. 30, le même jour, deux coulées se produisaient au lieu dit les «Pigettes ».

A la suite de quoi, le 13 également, se tenait, sous la présidence de M. Ie Préfet de la Savoie, une conférence à laquelle participaient des représentants de l'E.D.F., de l'Entreprise Industrielle, des Eaux et Forêts, des Ponts et Chaussées, de l'Armée, de la Gendarmerie et du Conseil municipal, représenté principalement par M. le Maire de Tignes.

Sur la demande de M. le Préfet, l'Administration des Eaux et Forêts était chargée de délimiter les zones réputées dangereuses; elle envoyait sur place un agent technique des Eaux et Forêts pour contrôler les chutes de neige, ainsi que pour relever les températures.

Ces résultats devaient être communiqués à M. l'Ingénieur Grivaz, des Eaux ef Forêts de la Conservation de Chambéry. Celui-ci devait proclamer l'état d'alerte dans les zones délimitées si cela s'avérait nécessaire. Un représentant de l'E.D.F., moi-même, représentait l'ingénieur GriYAZ sur place.

A cette réunion, il était décidé également de consulter à Davos (Suisse) les savants de l'Institut Fédéral de la Neige et des Avalanches, qui, sur la demande de E.D.F., enverraient deux techniciens pour prêter leur concours au futur Service de Détection des Brévières et indiquer les méhodes et le matétiel nécessaires.

Le 17 février, après une dernière entrevue avec les représentants d'E.D.F., des Eaux et Forêts et de l'Entreprise Industrielle, les zones à évacuer en cas d'alerte étaient définitivement arrêtées. Je ne m'étendrai pas sur tous les détails : qu'il suffise de savoir que le secteur des Brévières comportait trois zones : zone $A$, zone $B$, zone $\mathrm{C}$, qui, dans leur périmètre, englobaient un certain nombre de battiments ateliers, et beaucoup d'habitations particulières, ainsi que des dortoirs et cantines.

Dans l'après-midi, deux techniciens suisses, MM. Sroucky ef Dick, venaient aux Brévières initier les futurs nivologues à la lourde tâche qu'ils auraient à remplir.

I, Entreprise Industrielle mettait alors à notre disposition deux bons slieurs pour faire les observations en haute altitude.

Après deux jours de démonstration sur le terrain (profils de rupture stratigraphique, essais de déclenchement artificiel, etc.), les ingénieurs suisses nous quittaient, non sans avoir au préalable confié à M. I'Ingénieur Grivaz et moi-même le secret (si j'ose dire) de la détection.

De quoi se composait donc le Service de Détection au point de vue personnel? des techniciens suivants :

1 ingénieur des Eaux et Forêts à Chambéry, -.. 1 ingénieur de E.D.F. sur place.

- 1 agent technique des Eaux et Forêts, sur place. 
- 2 skieurs de l'Entreprise Industrielle, sur place également.

qui devaient surveiller :

\section{Zones a surveiller :}

- 3 couloirs d'avalanches, dont 1 surtout (ceIui de la Sache),

et qui avaient à leur disposition le matériel cidessous :

\section{Matériel employé :}

1 balance romaine.

1 thermometre maxima ef minima (type commercial).

1 cuve à neige.

1 lable à neige.

1 perche à neige,

complété plus tard, au 10 mars exactement, par: du matériel suisse, des dynamomètres de $10 \mathrm{~kg}$, $5 \mathrm{~kg}, 1 \mathrm{~kg}$, étriers et cylindres de prise de densité fabriqués à Bourg-Saint-Maurice.

\section{Méthode employée :}

C'est la méthode suisse, ou, si vous roulez, la

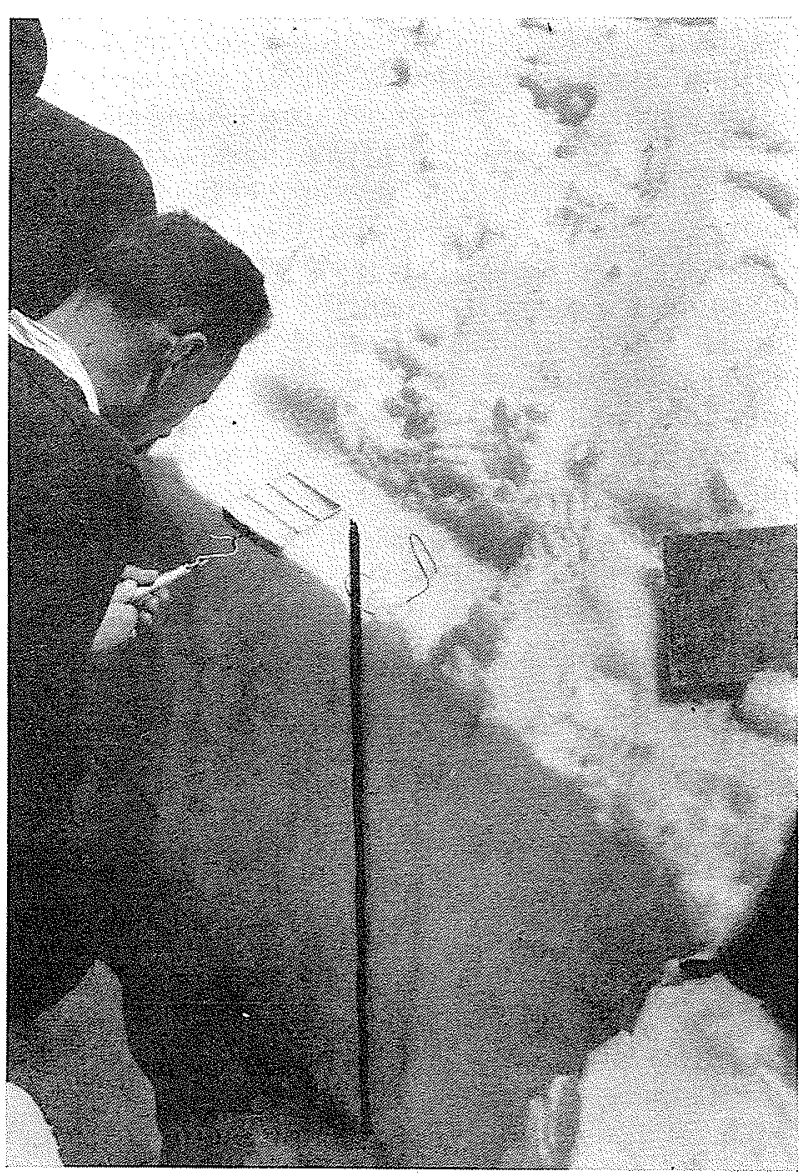

Fig. 1. - Mesures au cisamliement.

Cing mesures sont effectuées tous les deux jours, et le plus près possible des zones de ruptures présumées.

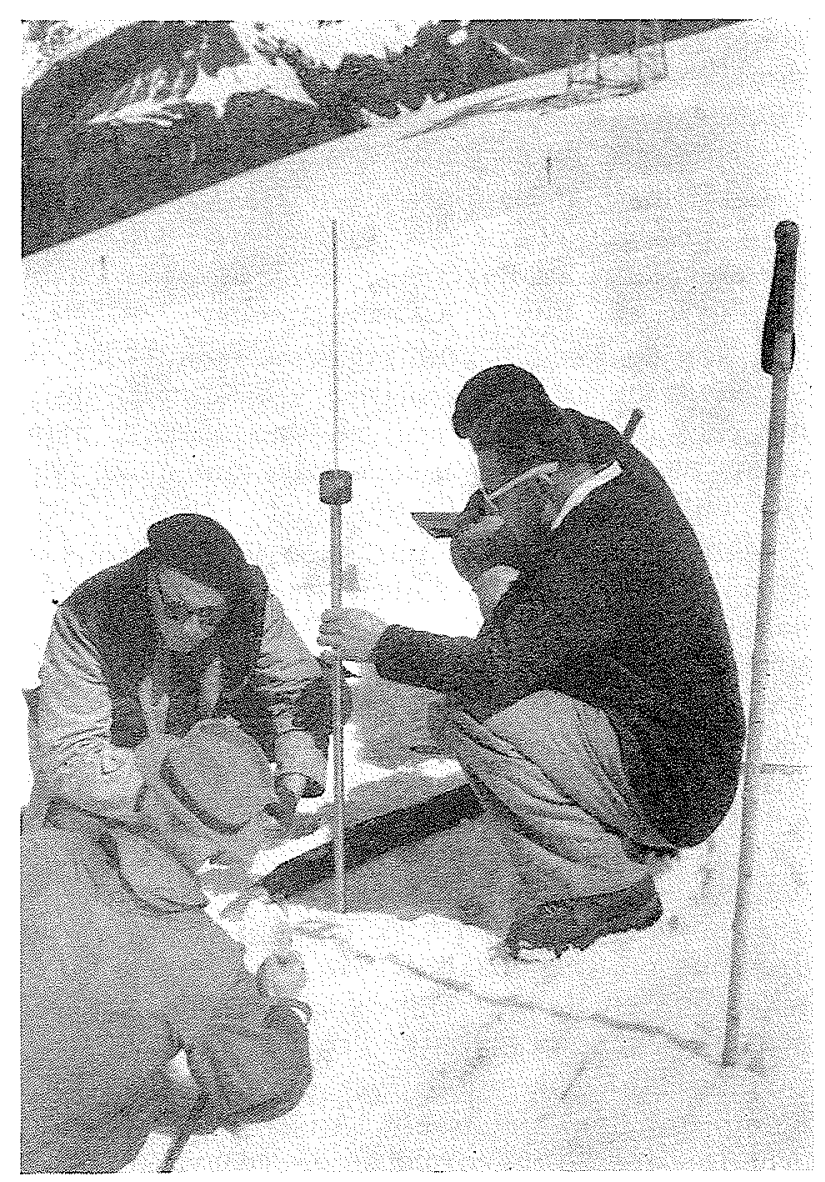

Fig. 2. - Soxdace aU batratie au Saloisle.

détermination de l'indice de rupture d'un dm² de neige. Pour cela, on creuse une tranchée jusqu'au sol, puis apres avoir rectifié bien verticalement le coté amont de la fosse, on dispose, sur un espace de neige soigneusement rafratchie, un appareil (sorte d'étrier) de $1 \mathrm{dm}^{2}$ que l'on enfonce sur toute son épaisseur dans la neige. A l'aide d'un dynamometre, on mesure le coefficient de rupture de $1 \mathrm{dm}^{2}$ de neige en exercant un effort de traction sur le $\mathrm{dm}^{2}$ en aluminium. Cette opération est répétée tous les 5 ou $10 \mathrm{~cm}$ suivant la continuité granulométrique plus ou moins grande des couches de neige. Puis la température de la neige est prise, d'abord en surface, puis de $20 \mathrm{~cm}$ en $20 \mathrm{~cm}$ et au sol.

On étudie également la cristallographic de chaque couche pour voir la grosseur des grains de neige et leur degré de métamorphose.

On termine par une prise de densité dans chacune des couches de neige repérées dans la tranchée.

Ces résultats sont consignés par un graphique que je vous montrerai tout à l'heure et, à l'aide d'un calcul spécial, on détermine s'il y a danger, ou non, de glissement.

Nous n'avons pu, faute de matériel, faire de 
sondages au battage, sauf quelques-uns cependant, exécutés à la fin de la campagne, grâce à l'obligeance de M. SUlzLÉE qui a mis une sonde de battage à notre disposition.

Cette méthode, apportée par les Suisses, avait déjà fait, au cours des hivers précédents, ses preuves, dans les Pyrénées, où M. SurzLté l'avait employée sur une très vaste échelle.

Grâce également aux conseils techniques de M. SulzléE, sa connaissance approfondie de la neige, son entrainement en la matière, nous avons pu mener à bien le Service qui nous avait été confié.

Malheureusement, il faut bien le dire, nous ne disposions pas de personnel ni de matériel suffisants pour nous permettre d'affirmer avec certitude s'il y avait réellement danger ou non.

Aussi, quand l'on songe que ce Service de Détection a été créé en un laps de temps très courl, équipé de matériel jusque-là inconnu, mis entre les mains diun personnel encore inexpérimenté, on voudra bien me comprendre et m'excuser si parfois, faute de renseignements indispensables, dus toujours au manque de moyens, j’ai dû prendre sous ma responsabilité de proclamer telle ou telle zone en danger alors que le danger n'était peut-être pas immédiat?

Il ne faut pas jouer non plus avec des éléments qui nous dépassent. Aussi valait-il mieux prendre une grande marge de sécurité que de ne rien faire dn tout et de risquer le pire.

Ainsi donc, avec de sobres moyens, un service organisé rapidement, nous avons terminé l'hiver sans aucun accident corporel ou matériel sur nos chantiers, sauf peut-ètre pour le personnel du Service Avalanches lui-même.

Nous devions souvent monter faire nos observations à $2.000,2.400$, alors que les Brévières, ne l'oublions pas, sont à 1.560 ; nous avions à traverser parfois trois ou quatre couloirs d'avalanche sans compter ceux de moyennes coulées. Songez un peu au temps et à l'effort qu'il faut exiger d'une équipe pour assurer avec le maximum de conscience un tel travail tous les deux jours, sinon tous les jours.

Quand nous n'étions pas sûrs de l'évolution du temps ou que celui-ci changeait brusquement sur le soir, comme cela arrive fréquemment en montagne, nous étions encore obligés de monter la nuit faire de nouveaux profils.

A titre documentaire, les heures du Service Avalanche, du 17 février au 10 mai, sont les suivantes :

844 heures qui se résument ainsi : en dehors des heures de travail où ce service pourrait etre considéré comme normal, le Service Avalanches a assuré 260 heures dont 216 de jour et 44 heures de nuit.
Le travail exécuté a été le suivant :

- 75 profils dont 20 au-dessus de $1.500 \mathrm{~m}$.

- 29 - $\quad$ - $\quad$ - $\quad 1.800 \mathrm{~m}$.

- 15 - - $\quad 2.000 \mathrm{~m}$.

- 11 dans diverses zones aux fins de contrôle.

- 17 reconnaissances en haute montagne.

Il a été enregistré pour l'agglomération seule des Brévières 4 avalanches de plaques de neige prévues vingt-quatre à trente heures à l'avance.

Si je livre ces chiffres, Messieurs, à vos réflexions, ce n'est pas pour essayer d'en tirer une gloire pour nous-mêmes ou pour le service que nous dirigions, mais simplement pour vous montrer à quel point la tâche est rude et dangereuse dans nos régions, ou si vous aimez mieux, comme je vous l'ai dit tout à l'heure, combien le problème est vaste ct digne d'intérêt.

En effet, la protection du chantier des Brévières n'est qu'une partie d'un problime d'ensemble qui intéresse les Alpes tout entières et d'autres régions de France où sont installés d'autres chantiers.

Le Service de Détection terminait sa mission le $10 \mathrm{mai}$

L'Electricité de France et les entreprises travaillant sur le chantier venaient, par cette première expérience, de comprendre à quel point un Service de Prévisions d'Avalanches, pour l'hiver prochain, pourrait leur rendre service. En effet, pour ne parler que des chantiers hydro-électriques de haute montagne, il est absolument indispensable d'assurer la sécurité du personnel travaillant dans des conditions souvent anormales et pénibles, durant certains hivers comme celui que nous avions traversé, par exemple, et qui, notons-le bien en passant, n'était pour la Savoie qu'un hiver moyen.

Sans vouloir en quoi que ce soit faire état d'un sentiment quelconque, en dehors du sentiment purement humain, il faut encore une fois que ceux, qui de par leur situation, sont amenés à travailler à $1.500 \mathrm{~m}, 1.800 \mathrm{~m}$., $2.000 \mathrm{~m}$ et même plus, n'aient pas pendant les hivers rigoureux le souci de leur protection propre ainsi que celle de leur famille. Car vous serez d'accord avec moi qu'un personnel qui se sent en sécurité fournira un rendement supérieur à celui qu'il donnerait s'il était obsédé continuellement par la présence d'un danger qui le menace. Cela, les Administrations intéressées l'ont compris et déjà, j'étais chargé de mettre sur pied un Service de Détection pour l'hiver prochain - service qui devait opérer avec des moyens plus puissants.

Après plusieurs entrevues avec M. Sulzlére qui a été pour moi un auxiliaire très précieux et très dévoué, j'élaborais un plan pour créer le Service qui allait fonctionner cet hiver.

Malheureusement, le choix d'un persomnel 
supplémentaire, les lenteurs apportées dans les commandes de matériel, n'ont pas permis l'entrée en fonctionnement de ce Service au $1^{\mathrm{er}}$ novembre, comme je l'avais prévu. Je n'ai pu commencer à faire les observations qu'à partir du $1^{\mathrm{er}}$ janvier 1951 , alors que $70 \mathrm{~cm}$ de neige étaient déjà tombés - ainsi nos observations risquaient d'être sérieusement compromises. Cependant, nous avons pu, malgré tout, rattraper le temps perdu.

A la suite de ces considérations générales, peutetre trop longues, mais nicessaires cependant, pour vous faire comprendre encore une fois l'ampleur du problème --.- considérations générales qui n'ont de technique que le sens qu'on veut bien leur donner, je vous parlerai de la création du Service de Détection qui a fonctionné cel hiver, et je m'efrorcerai de vous démontrer par un exemple typique que la détection en matière d'avalanches est désormais techniquement possible.

\section{SERVICE DE DETECTION 1950-51}

Ce service comprend :

PERSONNEI :

1 chef de service.

1 agent technique des Eaux et Forêts, comme adjoint.

2 observateurs skieurs (moniteurs de ski).

1 radio-secrétaire et dessinateur.

1 observateur météorologiste.

\section{LOGEMENT :}

1 bureau central.

1 salle de dessin et d'entrepôt du matériel.

1 chambre de veille.

MATÉrIEL vTrusé : 2 stations météorologiques. a) l'une à l'altitude 1.850 , comprenant :

--- 1 abri norvégien type haute montagne

- 1 héliographe.

- 1 thermomètre enregistreur.

- 1 thermomètre maxima.

- 1 thermomètre minima.

- 1 psychomètre.

- 1 hygromètre.

- 1 thermomètre étalon (lype « neige » gradué de $+10^{\circ}$ à $-20^{\circ}$ cenligrade)

- 1 anémomètre à main électro-magnétique papillon du modèle O.N.M. type "Météo »:

- 1 sonde de battage (modele suisse) servant à mesurer l'enfoncement initial de la neige, d'où la cohésion en surface.

- 1 table à neige.

- 1 tablette à neige.

- 1 cuve à neige.

- 1 balance romaine.

- 1 perche à neige. b) L'autre situće à proximité du Bureau Central, à l'altitude de 1.550 , comprenant:

- 1 abri norvégien du même type que le premier.

- 1 thermometre enregistreur.

- 1 psychomètre.

- 1 thermometre maxima.

-... 1 thermometre minima.

- 1 tablette à neige.

- 1 télévent à lecture directe de la vitesse du vent en mètre/seconde et de la direction du vent, que l'on obtient au moyen de voyants lumineux que l'on éclaire par le jeu d'une magnéto.

LAISON GNTRE LA STATION MÉtÉnologroue Supterieyne et Strtion Centrale

Celte liaison est assurce de deux facons :

- Par la transmission de messages météorologiques par radio.

- Par la transmission de messages par téléphone.

Les postes radio servent aussi en cas de coupures du téléphone, ce qui arrive malgré tout assez fréquemment. D'autre part, les messages restent ainsi la seule propriété du Service de Détection Avalanches (ce qui est extrêmement important) et ne sont pas court-circuitées, si j'ose dire, au risque d'être mal interprétés par des tiers, ce qui pourrait en effet avoir des conséquences très graves.

De plus, une liaison étroite est assurée égaJement avec la Station Météorologique de BourgSaint-Maurice, dont le chef de station, M. PLAsse, mis à notre disposition par la Direction de l'O.N.M., nous a rendu d'inappréciables services pendant cet hiver.

\section{Fonctonnement du Centre}

Météorologie. - Chaque jour à 8 heures, 11 heures et 17 heures, les messages météorologiques sont communiqués à la Station Centrale installée aux Brévières et comprennent les renseignements suivants :

Température au thermomètre enregistreur.

Température maxima.

Température minima.

Température au thermomètre sec du psychomètre.

Température au thermomètr mouillé.

Indication de l'hygromètre.

Vitesse du vent en $\mathrm{m} / \mathrm{s}$ et direction.

S'il y a lieu :

Hauteur de chutes de neige sur la table.

Poids de la neige dans la cuve.

Densité.

Durée de la chute. 
Hauteur totale de chute à la perche à neige.

Enfoncement initial de la sonde.

Ces renseignements sont consignés dans un livre de bord qui est tenu à jour par le secrétaire radio.

Parallèlement à ces relevés, à l'altitude 1.560 , sont relevés les appareils de la Station Basse, également aux mêmes heures. Leurs renseignements sont inscrits dans le carnet d'observation de la Météorologie Nationale.

a) Température. - Les températures maxima et minima sont ensuite représentées graphiquement, ainsi que l'écart de température enregistrée dans la journée.

b) Humidité. - Le pourcentage d'humidité relevé dans la journée est représenté graphiquement, ainsi que la différence entre le degré d'humidité du matin et celui du soir.

c) Isotherme. - L'isotherme du matin et celui du soir sont également traduits sur un graphique.

d) Neige. - Les graphiques « neige » traduisent les chutes de neige relevées à la table à neige - les données pendant le temps de chute déterminé, et le relevé, à la perche à neige, de l'enneigement total.

e) Profils. - Deux graphiques, I'un concernant la rive droite, l'autre la rive gauche, donnent l'interprétation des profils exécutés. Une courbe représente l'évolution du $\beta_{s}$ mesuré (ou si vous voulez, l'indice de rupture calculé au dynamomètre), l'autre le (i sin poids d'une certaine épaisseur de neige multiplić par le sinus de la pente.

L'état nivométrique de la neige est donné en fonction de ces deux courbes.

Deux graphiques, dits graphiques généraux, l'un rive droite, l'autre rive gauche, mentionnent le profil typique de chaque semaine, et de plus :

- La température maxima.

- La température minima.

- L'enfoncement initial de la sonde.

- La vitesse du vent.

- La direction du vent.

- Les avalanches constatées.

- L'humidité.

Les variations subies dans le manteau de neige sont aussi notées au jour le jour, et par là même, le danger ou non d'avalanches, en tenant compte de la résultante du $\beta_{*}$ mesuré et du sinus de la pente.

La différence entre le $\beta_{s}$ mesuré est portée en plus ou en moins sur un autre graphique. Si la différence est positive, autrement dit si le $\beta_{s}$ mesuré est plus grand que le $G \sin \varphi$, il n'y a pas danger, le degré de cohésion étant plus fort.
- Dans le cas contraire, il y aurait danger $\left(G \sin \varphi>\beta_{*}\right.$ mesuré).

- Si la différence est égale à 0 , il y a équilibre, d'où possibilité peut-être d'envisager le déclenchement artificiel. Malheureusement, a tort peut-être, les avis sont très partagés là-dessus.

En tenant compte du $\beta_{s}$ mesuré, du sinus de la pente et du poids de l'épaisseur de neige que nous avons étudiée, on en tire la formule :

$$
\begin{aligned}
& \beta_{s} m=G \sin \vartheta \\
& \sin \varphi=\beta_{*} / G
\end{aligned}
$$

ce qui nous donne la pente limite au-delà de Jaquelle le danger peut exister.

Ce résultat est représenté sur un graphique de pentes, tenant compte du terrain (boisé, herbeux, rocailleux, sablonneux).

Il est bien entendu que le danger, s'il existe, est un danger théorique. Le danger réel ne peut être affirmé que si l'on tient compte de divers facteurs, tels que le terrain, les conditions météorologiques, plus un certain nombre d'inconnues que, seules, nos recherches peuvent nous permettre de résoudre en particulier les recherches faites dans les Pyrénées, si, tontefois, on nous donne les moyens nécessaires pour qu'elles soient continuées.

Après cet apereu sur la mise en place, et l'organisation du Service de Détection Avalanches et sur sa nécessité d'être, je vais vous donner un exemple qui vous on fera comprendre plus directement le fonctionmement.

II s'agit là d'un cas typique d'avalanche, pris entre de nombreux autres. Je me propose done de vous montrer comment l'évolution de la neige a été suivie au jour le jour, et a abouti finalement au déclenchement d'une avalanche; d'abord par profils stratigraphiques, puis par mesures nivométriques, enfin par l'étude des conditions météorologiques.

\section{Avalanche de plaques de neige sèche du 22 février 1951 à 15 h 20}

18 Fúvrier. - A dater de ce jour, la situation générale nivométrique en ce qui concerne le versant de la rive gauche de l'Isère, et plus spécialement le versant dit de la Carrière des Brévieres, pris comme exemple, se présentait de la facon suivante :

Au PoINT DE VUE NEIGE. - L'enneigement variait ce jour de $1,40 \mathrm{~m}$ à $1.600 \mathrm{~m}$, à $2,10 \mathrm{~m}, 2,40 \mathrm{~m}$ environ à $2.000 \mathrm{~m}$. Par suite du vent, la neige était surtout accumulée en grosse épaisseur dans les combes, les sommets étant recouverts d'une neige tôlée en surface. 


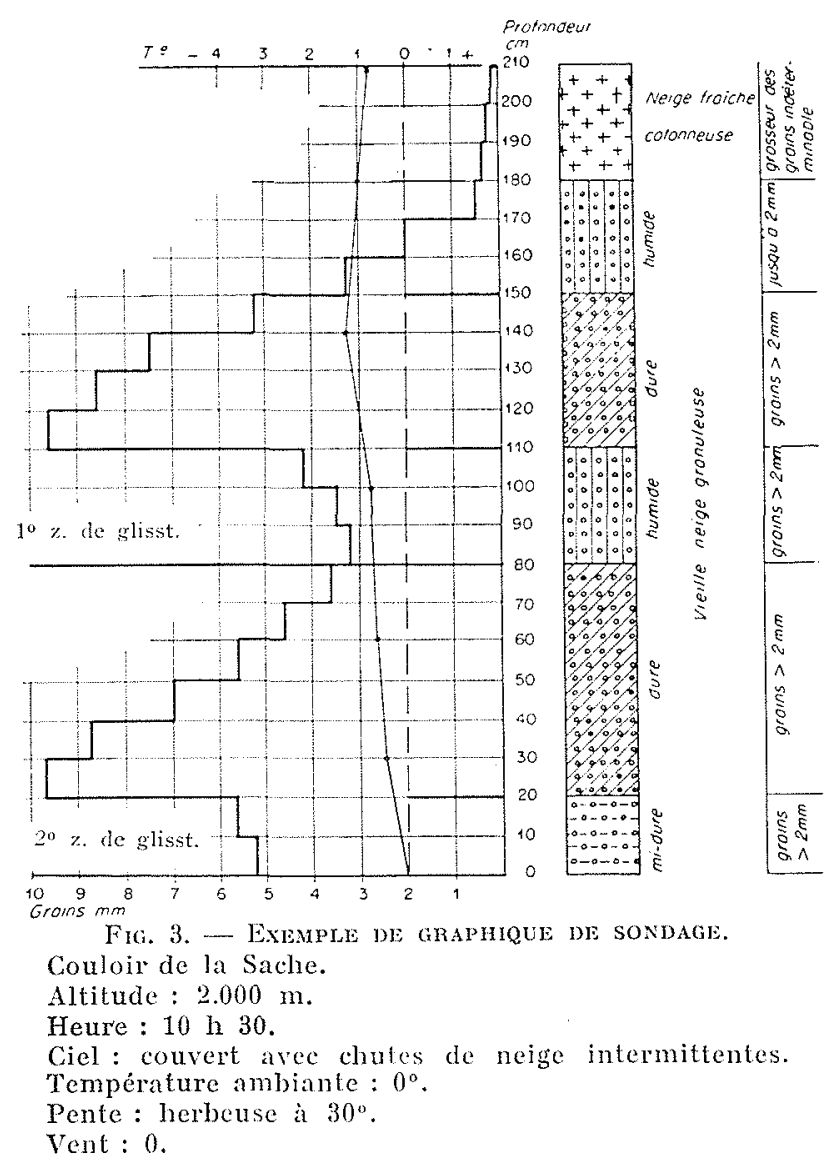

Le profil de neige à la rupture, ainsi que le profil stratigraphique ctablis ce jour même, se reproduisaient de la manière suivante (profil $n^{\circ} 33$ du S.D.A.).

Sur ce profil stratigraphique, on peut distinguer en gros trois couches de neige, la dernière englobant un certain nombre de couches intermédiaires mais à peu près de même nature.

$1^{\circ}$ Une couche supérieure de $30 \mathrm{~cm}$. Cetle couche est constituée de grains extrêmement fins, non métamorphosés, dont la grosseur est indéterminable (la grosseur des grains de neige est calculée en prenant un peu de neige sur un carré millimétré, en plexiglas ou en aluminium; à l'aide d'une loupe on peut aisément apprécier la grosseur d'un grain de neige). Cette couche est faite uniquement de neige fraîche.

$2^{\circ}$ Une couche inférieure de $30 \mathrm{~cm}$ de profondeur. Elle est alors formée par de la vieille neige humide à grains fins, très légèrement métamorphosés, la grosseur de ces grains ne pouvant être appréciée que très approximativement, au $1 / 2 \mathrm{~mm}$ (en effet, il est très difficile avec des grains si petits et enchevêtrés comme ils sont, d'en déterminer la grosseur exacte).

$3^{\circ}$ Une série de 4 couches.

Cet ensemble de couches part du sol jusqu'à $1,50 \mathrm{~m}$ de hauteur. Les 4 couches ont été facile- ment repérées sur le terrain, mais leurs éléments granulométriques étant sensiblement les mêmes, on peut facilement les confondre en une seule couche.

On trouve là de la vieille neige à métamorphose avancée - grains variant du millimètre et demi à $3 \mathrm{~mm}$. Au point de vue densité, la couche était sulfisante pour ofrir malgré tout une bonne cohésion.

\section{Mesures NuYométrioues :}

a) Densité :

La prise de densilé effectuće dans les différentes couches donnait les résultats suivants :

$1^{\text {ro }}$ couche, densite moyenne : 0,140 .

2" couche, densité moyenne : 0,220.

Puis, en dessous de $60 \mathrm{~cm}$ dans les autres couches, on avait le chiffre de 0,300 .

\section{b) Rupture:}

Les mesures dynamométricques donnaient alors une série de chiffres dont il faut retenir surtout les principaux, c'està-dire :

Un indice maximum de rupture allant de $0,300 \mathrm{~kg}$ à $0,500 \mathrm{~kg}$, avec augmentation progressive dans la deuxieme couche et par ailleurs dans les autres couches on relevait des indices variant de $3,100 \mathrm{~kg}$ à $3,500 \mathrm{lg}$ par $\mathrm{dm}^{2}$.

c) Calculs du $\beta_{s}$ mesuré et du $\mathrm{C} \sin \varphi$ :

Les calculs du $\beta_{8}$ mesuré et du $G \sin \varphi$ révélaient une stabilité totale de toute la partie inférieure du manteau de neige. En effet, on avait $\beta_{s}$ mesurć $:=1,500 \mathrm{~kg}$, $\mathrm{G} \sin \varphi=0,374$. La difrérence entre le $\beta_{\text {s }}$ mesuré et le $\mathrm{G} \sin 0$ était donc de $1,126 \mathrm{~kg}$, ce qui nous donnait un coefficient de sécurité très élevé. D'autre part, le calcul effectué indiquant sur quelle pente il pouvait y avoir danger, nous donnait un sinus «absurde», donc une grande sécurité également.

\section{Renseignements météorologioues :}

Au point de vue météorologique, le temps restait couvert les 18 et 19 février. Le plafond était relativement bas et le brouillard intense. L'état hygrométrique de l'air était de $95 \%$.

Les 21 et 22 février, le ciel se couvrait à nouveau de nuages. D'abondantes chutes de neige se produisaient le 18 et la hauteur de neige tombée sur la table donnait un chiffre de $37 \mathrm{~cm}$.

Dans la joùrnée du 19 on enregistrait $18 \mathrm{~cm}$

$$
\begin{array}{llll}
- & 21 & - & 16 \mathrm{~cm} \\
- & 22 & - & 20 \mathrm{~cm}
\end{array}
$$


Le vent malgré tout restait assez faible et soufflail de la direction du N.W. Sa ritesse ćtait environ de 2 à $5 \mathrm{~m} / \mathrm{s}$.

Température. - L'évolution de la température de l'air, relevée sous abri à la station de $1.800 \mathrm{~m}$ au Chevril, donnait les renseignements suivants :

\section{Maxima Minima}

$\begin{array}{llr}\text { Le } 18 \ldots \ldots & +2 & -6 \\ \text { Le } 19 \ldots \ldots & -1 & -8 \\ \text { Le } 20 \ldots \ldots & +2 & 5 \\ \text { Le } 21 \ldots \ldots & +3 & -5 \\ \text { Le } 22 \ldots \ldots & -3 & -5\end{array}$

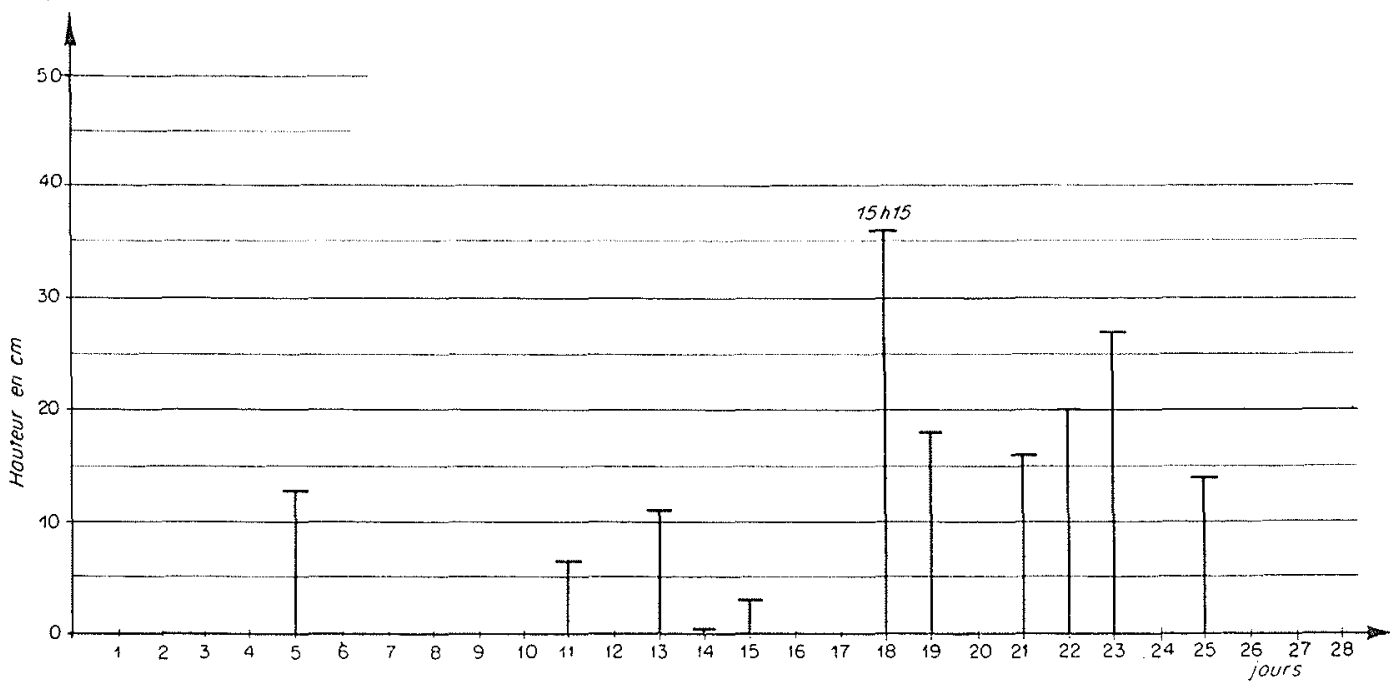

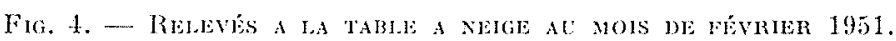

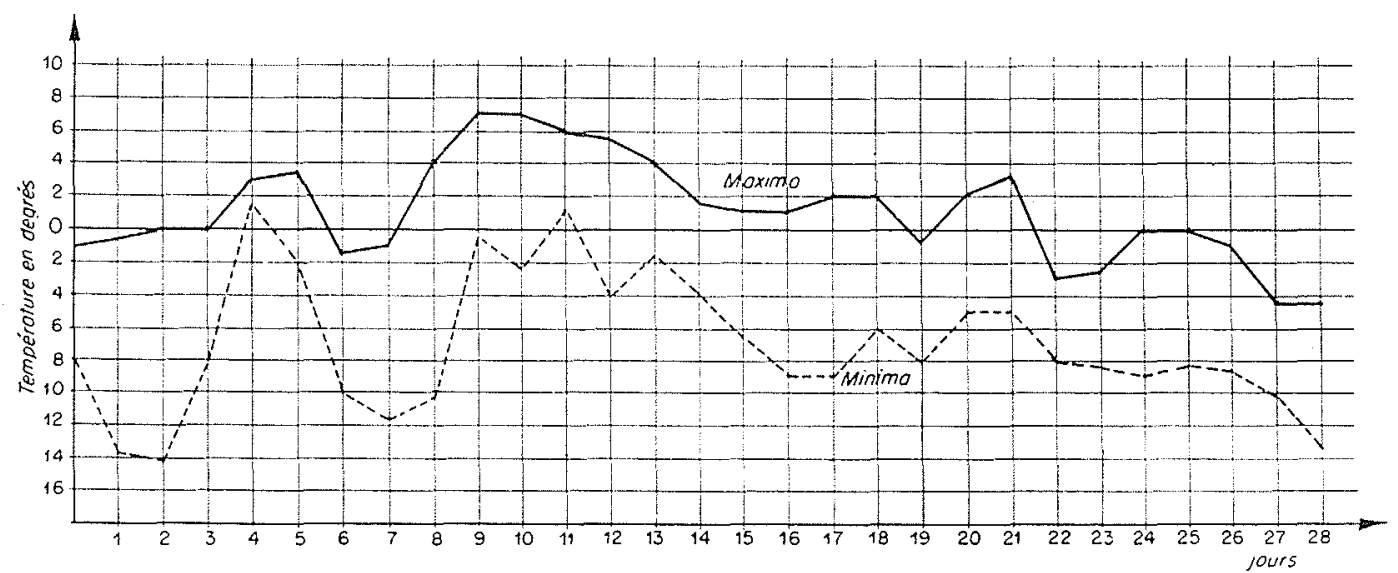

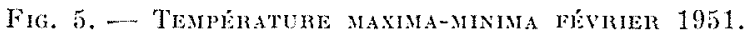

Nos graphiques indiquaient également un danger de plus en plus croissant, et nous nous trouvions déjà dans une période préavalancheuse, si j'ose dire.

Etabli le 17 février 1951 à 18 heures, le bulletin d'avalanches S.D.A. suivant était publié.

Station. - Les Brévicres.
Or la métamorphose de la neige entre dans sa phase la plus active à une température très voisine de $0^{\circ}$; par conséquent, étant donné les températures très basses enregistrées durant ces cing jours, en altitude, il n'existait qu'une métamorphose très lente de la neige nouvellement tombée. Cette neige restait par conséquent poudreuse et était prète à « fluer » à la première occasion.

De plus, bien que le vent soit faible, il agissait malgré tout d'une manière assez intense sur la neige fraîche. En effet, la structure interne du manteau de neige révélait toujours un état pulvérulent. 
la majeure partie du manteau de couverture. Dautre part, la densité de la neige varie de 0,300 à 0,400 .

Hauteur de neige moyenne : 2,20 $\mathrm{m}$ à $2.000 \mathrm{~m}$. Hygrométrie : $52 \%$ - - Vent : 0.

Température sous abri : à $17 \mathrm{~h}$ : +2 .

Etat de la neige: faible cohésion.

En efret, le 18 février fini à 13 h. 30, une coulée de neige très superficielle se déclenchait sur le versant de la Carrière. Cette coulée, formée de neige sèche sans cohésion, intéressait uniquement la couche supérieure de neige fraichement tombée (à titre documentaire, l'agent technique des Eaux et Forêts attaché à mon Service, et une autre personne se trouvèrent pris dans un tourbillon très violent, avec impossibilité de reprendre leur soulfle durant quelques instants). L'alerte passée, deux poteaux télégraphiones étaient couchés sur la route.

Cette coulée de neige, ainsi que celles qui se sont produites dans la mème journée, avaient été prévues dans le bulletin du Service de Détection Avalanches, $n^{\circ} 9$ S.I.A., comme vous venez de le voir.

Etant donné l'augmentation, de jour en jour, de la couche de neige frầche en montagne, et un abaissement de température, il ne pouvait y avoir aucune consolidation des couches nouvelles.

D'autre part, nos graphiques, qui indiquaient depuis quelques jours des risques d'avalanches sur des pentes de plus en plus faibles, nous signalaient maintenant un net danger d'avalanches, compte tenu toujours des accidents du terrain.

C'est ainsi que, dès le 19 février, on signalait ce danger d'avalanche :

Bulletin S.D.A. 10 établi le 19 février 1951 à $8 \mathrm{~h}$. Station. - Les Brévières.

Emplacement. - Couloir de la Sache (zone B), Carrière des Brévières. - La Raie. R.N. 202 Nouvelle.

La neige qui tombe depuis samedi 17 à $19 \mathrm{~h}$. s'est entassée sur les pentes et dans les combes. Le vent a accumule des masses instables qui n'ont pu, étant donné la température très basse, faire prise avec le manteau primitif.

De ce fait, le danger d'avalanches est grand sur les pentes citées ci-dessus. La neige poudreuse peut tres bien entrainer une grosse partic du manteau de neige primitif entré ces jours derniers dans une métamorphose avancée.

Mauteur de neige moyenne à $2.000 \mathrm{~m}=2,70 \mathrm{~m}$. Température sous abri : -5 .

Hygrométrie : $99 \%$.

Etat de la neige : aucune cohésion en surface, fable cohésion en profondeur.
Toutes ces pentes sont dangereuses pour le ski.

Le danger d'avalanches était à nouveau signalé dans un bulletin publié le 21 dont voici la teneur :

Bulletin S.D.A. 11. - Etabli le 21 février 1951 à 8 h. 30.

Station. - Les Brévières.

Emplacement. - Couloir de la Sache (zone B). - Carrière des Brévières. - La Raie. R.N. 202 Nouvelle F.O. 1-2-3-4-5.

La chute de neige actuelle et celle de ces jours derniers ont amené un gros danger d'avalanches. Le vent qui va se lever progressivement (foehn) augmentera encore le danger durant un certain temps. Il faudra autant que possible éviter de circuler sur la route des Boisses. L’avalanche de la Carrière des Brévières peut ètre dangereuse; également par la neige et par le souffle.

Température sous abri : -3 .

Enneigement moyen a $2.000 \mathrm{~m}: 3 \mathrm{~m}$.

Hygrométrie : $95 \% \mathrm{D}$ 'H 20.

Densité neige : 0,140 .

Comme vous le voyez, il y avait à ce moment un danger sérieux. Autrement dit, l'évolution de la neige, qui avait été suivie pas à pas, était entrée dans une phase ultime, ou l'équilibre des masses de neige devait etre rompu, sous l'elfet d'une force dynamique queleonque (le vent par exemple).

La chute de cette masse de neige pouvait très bien entrainer également non seulement la totalité de la neige fraiche tombée dans les vingtquatre heures précédentes, mais encore la neige plus ancienne provenant des chutes des cing aerniers jours.

Le graphique P 37, en date du 20 février 1951, dévoilait en effet :

une couche de neige fraiche de $45 \mathrm{~cm}$ avec grosseur des grains indéterminables, puis

deux couches de même nature d'une épaisseur de $35 \mathrm{~cm}$,

couches mi-dures,

une couche tendre de $15 \mathrm{~cm}$, enfin

une couche dure de $35 \mathrm{~cm}$ ave lamelle de glace intermédiaire.

On pouvait donc craindre un décollement de manteau jusqu'à la couche de glace rencontrée à $40 \mathrm{~cm}$ au-dessus du sol.

La temperature de la neige était la suivante:

- 5 en surface.

- 2 à $20 \mathrm{~cm}$ de la surface.

- 2 à $20 \mathrm{~cm}$ plus bas.

- 1,8 à $70 \mathrm{~cm}$.

-1 à $35 \mathrm{~cm}$.

et ensuite 0 . 
Jusqu'à $40 \mathrm{~cm}$ au-dessus du sol, la neige n'était pour ainsi dire pas entrée encore dans sa phase de floculation.

\section{Avalanche du 22 février à 15 h 20}

Dans la matinée du 22 février, la neige n'avait pas cessé de tomber, neige poudreuse, sans cohésion, d'une densité extrêmement faible $0,100 \mathrm{~kg}$ à $0,120 \mathrm{~kg}$ environ.
Les températures enregistrées à la Station du Chevril donnaient les résultats suivants :

$$
\text { Enregistreur Maxima Minima }
$$$$
\begin{array}{crccc}
\text { A } 8 \text { h. } \ldots \ldots & 7 & -8 & -3 \\
\text { A } 11 \text { h. } \ldots \ldots & -6,5 & -6 & -6 \\
\text { A } 14 \text { h. } \ldots \ldots & -6 & 6 & 5,5
\end{array}
$$

Le vent soufflait à $2.000 \mathrm{~m}$ à la vitesse de 6 à $7 \mathrm{mi} / \mathrm{s}$.

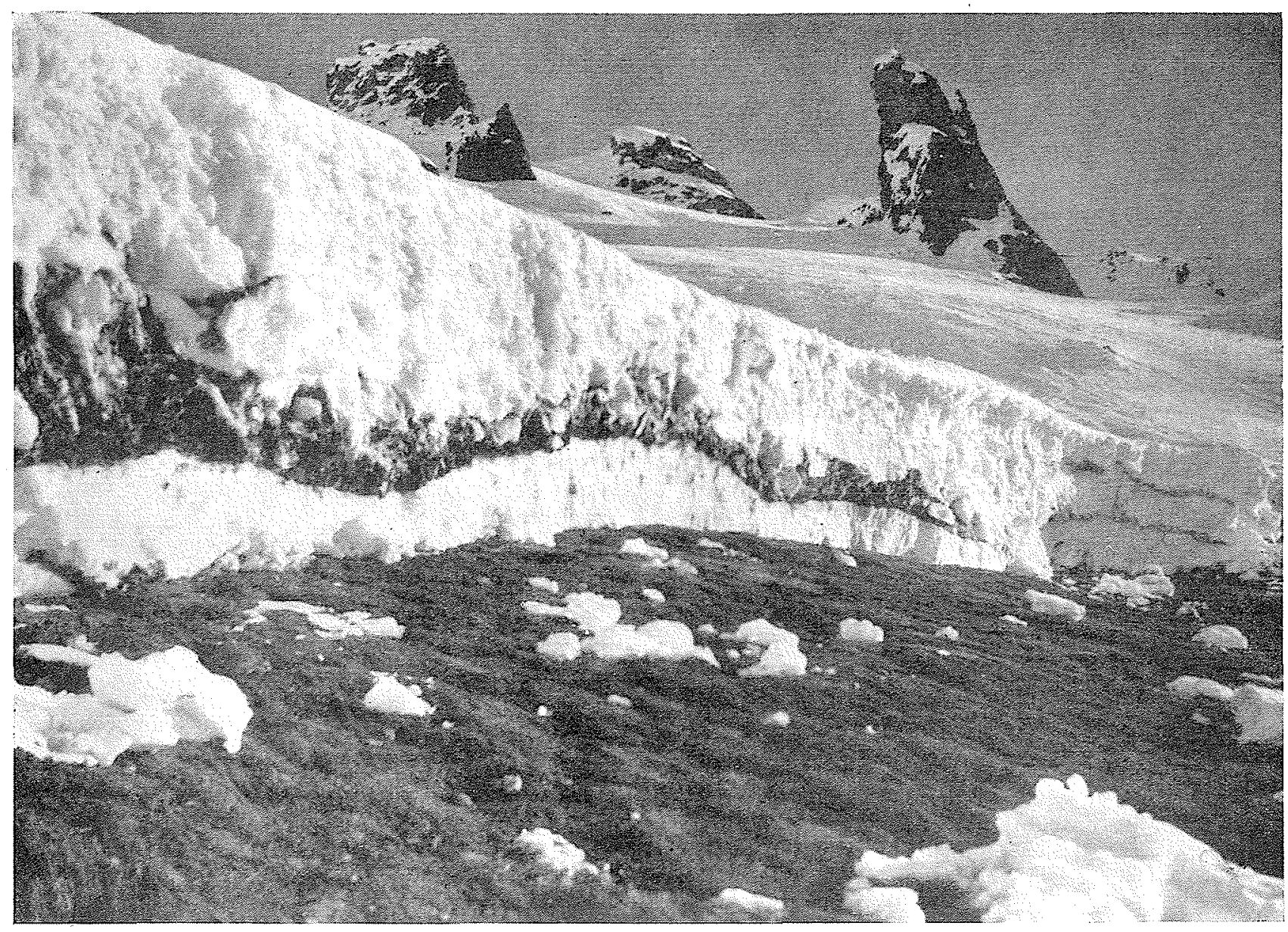

Fig, 6. - Photographe de ruptume d'avalanche.

La direction variait entre NE, SW et N.S..

A 15 h. 20, une avalanche de neige poudreuse se détachait de la cote $1.900 \mathrm{~m}$, au-dessus de la Carrière des Brévières, et dévalait la pente assez raide à cet endroit, provoquant un accident qui aurait pu avoir les plus graves conséquences.

Le souffle a duré environ 1 minute $1 / 2$, d'abord par rafales puis continu. On aurait pu évaluer le souffle à la vitesse de 90 à $100 \mathrm{~km}$ à l'heure, en se basant sur l'échelle de Beaufort (degré 10 de l'échelle).
Le cubage de cette avalanche étant de l'ordre de $2.000 \mathrm{~m}^{3}$ environ, le front s'arrêtait en face d'un dortoir, de bureaux et de cantines.

Fort heureusement, sur l'ordre du Service de Détection, toute précaution avait été prise et notamment les fenêtres de tous les bâtiments avaient leurs volets fermés, ce qui les préserva de dégâts infiniment plus sérieux.

Dégâts constatés. - Une seule fenêtre non munie de volets eut ses carreaux brisés. Le souffle de l'avalanche put ainsi s'engoufrer à la manière 
d'air comprimé, par cette ouverture qui lui était offerte. Le toit du bâtiment où est installé le Bureau central du Service de Détection et comportant une centaine de personnes environ, fut soulevé tout d'abord partiellement; puis, peu à peu, les ouvertures s'agrandirent et sous l'effet des masses d'air considérables contenues dans le local et qui trouvaient leur entréc de partout, la toiture fut finalement arrachée sur une surface de $200 \mathrm{~m}^{2}$ environ.

D'autres bâtiments avaient également subi des dégâts importants et au total $850 \mathrm{~m}^{2}$ de toiture durent être refaits. Il faut signaler également l'obstruction de la R. N. 202 sur laquelle il n'y eut pas d'accident de personnes, la circulation y ayant été très réduite depuis la veille, sauf en cas d'urgente nécessité.

Les fils téléphoniques et électriques jonchaient la route et les bâtiments sur une distance de $800 \mathrm{~m}$. L'antenne Radio du Service Détection ćtail gravement endommagée. Nous avons dû à coups de piolets dégager nos appareils météorologiques de l'abri, et si nous avions pu mesurer l'effort de cisaillement de cette neige ainsi comprimée, nous aurions trouvé des chiffres de l'ordre de 30 à $38 \mathrm{~kg}$.

Le même jour, à 4 heures du matin, une avalanche de neige poudreuse emportait $120 \mathrm{~m}^{3}$ de bois (surtout par son souffle) au lien dit : « La Gurra $\Rightarrow$ (près de la Savine).

Une autre avalanche faillit cmporter un groupe de maisons au lieu dit « Le Chapuis » (près de Sainte-Foy-en-Tarentaise).

Les jours suivants, d'autres avalanches se produisirent dans toute la valléc, mais sans gros dégâts, par suite des mesures de sécurité envisagées des la publication de nos bulletins.

Le 24, nous publions un autre bulletin d'avalanche S.D.A. 12 qui a confirmé par la suite nos prévisions.

Etabli le 24 février 1951 à 9 heures.

Station. - Les Brévières.

Emplacement. - Couloir de la Sache (zone B). - Carrière des Brévières. -- R.N. 202 Nouvelle. - La Raie. - Fenêtre 0 à 14.

L'abondante chute de neige de ces jours-ci a entassé $1,40 \mathrm{~m}$ de neige sèche, notamment dans les combes où le vent a accumulé des masses importantes de ce matériau.

Le danger d'avalanches de neige sèche est toujours grand, mais en cas de déclenchement de celles-ci le souflle sera moins important du fait de l'humidité aecrue de la neige par suite de l'élévation de température. Par contre, cette neige ayant augmenté de densité, ces avalanches, sous l'action de la hausse de temperature et du vent qui va s'élever progressivement et qui va sévir durant quelques jours, peuvent entraîner une grosse partic du manteau de neige, créant aussi des avalanches de fond qui seront plus dangereuses par la neige mème que par le souffle. Ces avalanches seront tres conséquentes, notamment en dessous de $1.300 \mathrm{~m}$. Les fenêtres 0 à 14 peuvent subir de telles avalanches.

Enneigement a $2.000 \mathrm{~m}: 3 \mathrm{~m}$.

Température sous abri : - - 4,5.

Hygrométrie : $98 \%$.

Densité de la neige : 0,200 .

\section{Conclusion}

A la suite de cet exemple caractéristiquo où je vous ai montré comment on suit l'évolution des couches de neige grâce à des moyens techniques puissants, on constate que c'est par une étude sérieuse du manteau de neige, par une connaissance approfondie des lois de la nivologie, par une connaissance exacte des conditions météorologiques, et j'insiste sur ce point, que l'on a pu aux Brévières, dans ce cas, comme dans tous ceux où le Service de Détection a dû intervenir, prévoir avec une quasi certitude Je danger qui menacait le chantier.

Et en terminant, je suis affrmatif, et M. SulzLÉE l'est autant que moi, pour dire qu'actuellement, si loutes les conditions que je viens de citer sont remplies, la prévision d'avalanche est maintenant du domaine du possible et ce serait une grave imprudence que de ne pas l'admettre, car l'on serait coupable en laissant ainsi sans défense des chantiers, des stations, des routes qui pourraient être protégés d'une manière efficace par un Service de Détection d'Avalanches. 